\title{
The effect of labels on short-term memory for colors with nursery school children'
}

HARLEY A. BERNBACH

CORNELL UNIVERSITY

Studies of short-term memory for non-verbal material with young children have yielded results qualitatively different from similar studies with adults. Introduction of labels, however, makes the children's performance very nearly like that of adults.

One of the common methods for studying shortterm memory (STM) is to present a short list of items and then probe for recall of one of them. The shape of the forgetting function thus obtained is exemplified by the results of a study by Phillips, Shiffrin, \& Atkinson (in press). They presented a series of cards bearing color patches to college student Ss who were then required to identify one of them. Figure 1 shows a typical short-term forgetting function, obtained when the presented series contained eight cards. There is perfect performance on the most recent item, the recency curve is S-shaped, and there is a marked primacy effect.

Atkinson, Hansen, \& Bernbach (1964) used a similar procedure to study STM in nursery school children. They presented eight animal cards to $S$, placed them face down in a row, and then showed a cue card identical to one of the eight on the table. S had to "find" the match to the cue card. Atkinson et al noted some qualitative differences between the forgetting function they obtained and the typical one for adults. As shown in Fig. 1, immediate performance was not so high, there was no S-shape, and the marked primacy effect was absent.

Some recent storage-based theories of STM suggest that factors related to rehearsal of individual items cause the S-shape and the primacy effect. Thus, one might hypothesize that the shape of the children's forgetting function was due to a failure to rehearseand such a failure might come about if they remembered the pictures as pictures rather than labelling them, since pictures would be difficult to rehearse.

The present study was designed to test this hypothesis by introducing labels for the stimuli into the Atkinson et al task and comparing the forgetting functions obtained from a label (L) and no-label (N) condition.

\section{Method}

The experiment involved a long series of discrete trials. On each trial, a display of either four, six or eight items was presented. A display consisted of a series of $2 \times 3-1 / 2$ in. cards with a $3 / 4 \times 1-1 / 2$ in. colored patch in the center. Four colors were used, deliberately chosen to be difficult for young children to label; they were blue-gray, turquoise, red-violet, and salmon. The cards were shown to $S$ one at a time, at a 2 sec. rate, and placed face down on the table in a row. In condition L, Ss named each card as it was presented; they were instructed to use the labels grey, green, pink, and orange, which were close to the actual colors. When the entire series had been presented, E pointed to one of the cards and asked $S$ to identify it by pointing to the appropriate color on a disk containing the four colors in a circular array. In condition $L, S$ was required to name the color as well. Each $S$ was run for 36 trials in two 18 trial sessions, and was rewarded with a raisin or candy after each trial. Each $\mathrm{S}$ was tested twice at each position in the three series. Across Ss, each color appeared equally often at each position.

Ss were four and five year olds enrolled in the Cornell University nursery school. In group L, the mean age was 60 months, $\mathrm{S}=3.8$ months, while in group $\mathrm{N}$, the mean age was 60 months and $\mathrm{S}=4.4$ months. There were 12 girls and eight boys in each group. Results

The results are summarized in Fig. 2 which shows the proportion of correct responses (CRs) pooled across the four, six and eight item lists, since the proportions of CRs were not significantly better than

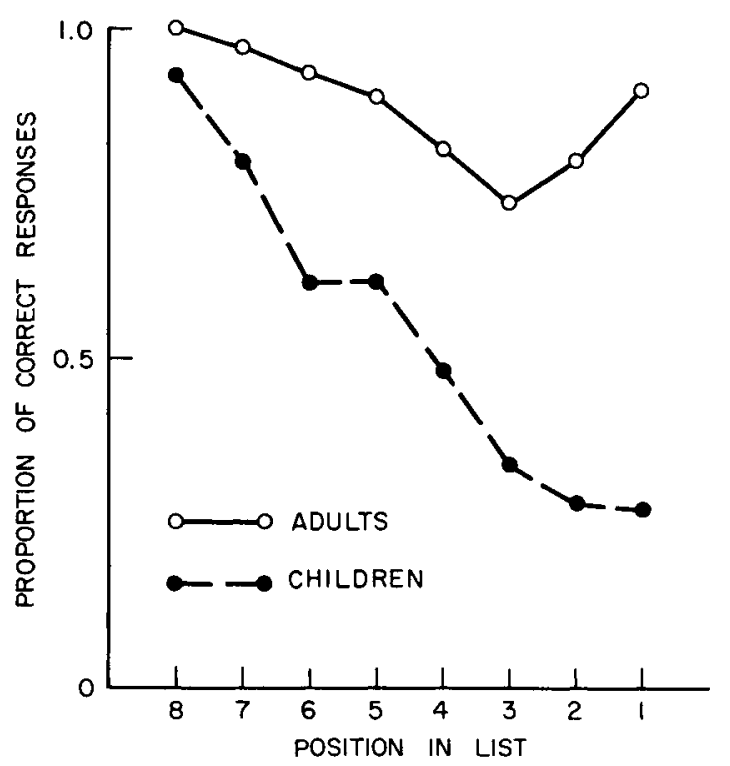

Fig. 1. Short-term forgetting functions for college students and young children. 


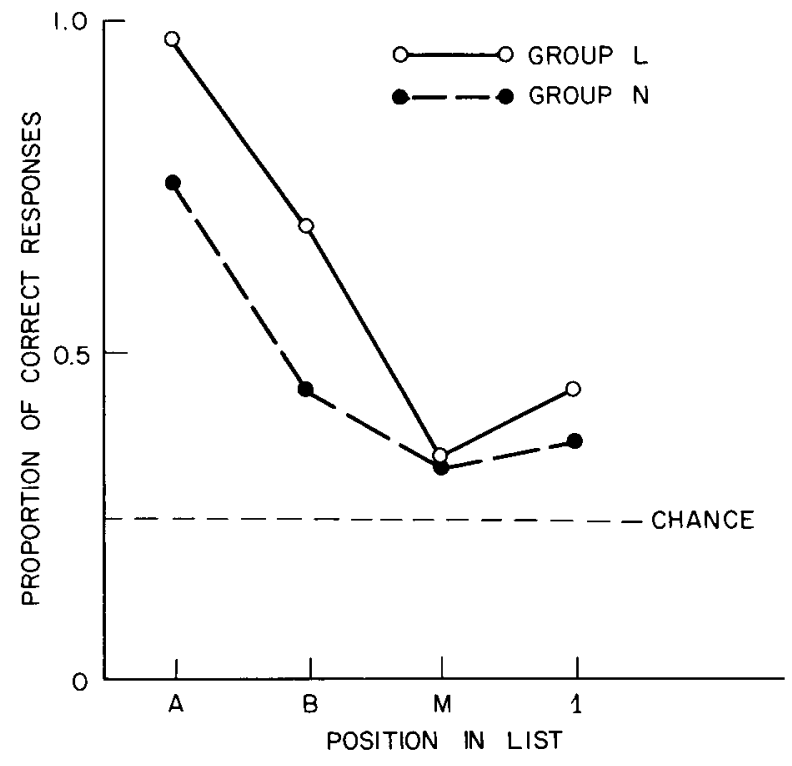

Fig. 2. Short-term forgetting functions with and without labels. $A$ and $B$ are the most recent and next most recent item presented, 1 is the first item, and $M$ is the mean for all other items.

chance for any items in the middle of the lists. The proportion of CRs is plotted as a function of retention interval; thus $A$ is the most recent item (last in set), $B$ the next most recent, and 1 the first item presented. The proportion of CRs pooled across all other items in the three series is at point $M$.

All points differ significantly from chance except $M$ (both groups) and 1 for group N. Points A and B differ significantly between groups, but point 1 does not.

\section{Discussion}

The extent to which the two forgetting functions of Fig. 2 resemble their respective prototypes is quite striking. Though quantitatively lower than the forgetting functions of college students in a similar task (Fig. 1), the group L forgetting function has all three of the common characteristics of these functions: near-perfect performance on the most recent item, a slight S-shape, and primacy. The group $\mathrm{N}$ curve, on the other hand, looks like the Atkinson et al datai.e., a simple exponential function.

The results are consistent with a rehearsal hypothesis if it is assumed that Ss rehearse only those items that they label, and that adult Ss tend to label stimuli such as colors and animal pictures automatically. Rehearsal of early items will produce primacy due to the greater opportunity to rehearse earlier items in the list, and rehearsal will also cause a greater effective degree of learning leading to a more shallow forgetting function at the recent positions. Further support for this argument comes from the similar recency result of Brelsford \& Atkinson (1966) for forced rehearsal in a continuous task.

At any rate, it appears that the qualitative difference between the STM performance of children and adults that was observed by Atkinson et al may be traced to the effects of labelling on STM, rather than to any inherent difference in the memory processes of children and adults.

\section{References}

Atkinson, R. C., Hansen, D. N., \& Bembach, H. A. Short-term memory with young children. Psychon. Sci., 1964, 1, 255-256. Brelsford, J. W., \& Atkinson, R. C. Recall of paired-associates as a function of overt and covert rehearsal procedures. Paper presented at Psychonomic Society, St. Louis, October 27, 1966.

Phillips, J. L., Shiffrin, R. M., \& Atkinson, R. C. The effects of list length on short-term memory. J. verbal Learn. verbal Behav., in press.

\section{Note}

1. This research was supported in part by funds from the Cooperative Research Program, U. S. Office of Education. 